\title{
Ramos colaterais viscerais da artéria aorta abdominal em Myocastor coypus (nutria)
}

\author{
The collateral visceral branches of the abdominal aorta in Myocastor coypus (nutria) \\ Paulete de Oliveira Vargas Culau ${ }^{1}$, Rodrigo Cavalcanti de Azambuja ${ }^{2} \&$ Rui Campos ${ }^{1}$
}

\begin{abstract}
RESUMO
A nutria é um roedor originário do extremo sul da América do Sul explorado comercialmente por sua pele e carne. Neste trabalho, sistematizou-se os ramos colaterais viscerais da aorta abdominal. Utilizaram-se 30 nutrias, 15 fêmeas e 15 machos, com o sistema arterial aórtico-abdominal preenchido com látex 603. A aorta abdominal emitiu ventralmente como ramo colateral visceral à artéria celíaca, que se trifurcou nas artérias gástrica esquerda, hepática e lienal. Os ramos da artéria celíaca promoveram a irrigação do estômago, duodeno, fígado, pâncreas e baço. A seguir, a aorta abdominal emitiu a artéria mesentérica cranial, de calibre maior que a artéria celíaca, justaposta caudalmente. A artéria mesentérica cranial emitiu ramos, vascularizando a parte final do duodeno, lobo direito do pâncreas, jejuno, íleo, ceco, cólon maior e cólon menor. A aorta abdominal emitiu lateralmente as artérias renais direita e esquerda. As artérias adrenais foram ramos colaterais diretos das artérias renais e/ou da artéria frênico-abdominal. A artéria mesentérica caudal foi emitida da superfície ventral da aorta abdominal, próximo à sua bifurcação em artérias ilíacas comuns. A artéria mesentérica caudal lançou ramos para o cólon menor e porção cranial do reto. Os ramos colaterais viscerais diretos da aorta abdominal foram: artéria celíaca, artéria mesentérica cranial, artérias renais e artéria mesentérica caudal. Os ramos viscerais indiretos foram: artérias adrenais e artéria umbilical. A artéria ilíaca externa emitiu a artéria umbilical que irrigou a bexiga, e continuou-se como uma grande artéria uterina, nas fêmeas, irrigando todo o útero, inclusive os ovários e, nos machos, a artéria testicular, que irrigou o testículo.
\end{abstract}

Descritores: artérias abdominais, vascularização, roedor, nutria, Myocastor coypus.

\section{ABSTRACT}

Nutria is a native rodent from the south of South America commercially explored for meat and fur. In this paper the collateral visceral branches of the abdominal aorta were systematized. Thirty nutrias were used, 15 males and 15 females, with the aortic-abdominal arterial system injected with latex 603. The abdominal aorta emitted, ventrally, as visceral collateral branch the celiac artery, which trifurcated in left gastric, hepatic and lienal arteries. The celiac artery branches promoted the irrigation of the stomach, duodenum, liver, pancreas and spleen. Following, the abdominal aorta emitted the cranial mesenteric artery, of bigger caliber than the celiac artery, caudally juxtaposed. The cranial mesenteric artery emitted branches to vascularizing the final part of the duodenum, right lobe of the pancreas, jejunum, ileum, cecum, large colon and small colon. The abdominal aorta laterally emitted the right and left renal arteries. The adrenal arteries were direct collateral branches of the renal arteries and/or the frenic abdominal artery. The caudal mesenteric artery was emitted from the ventral surface of the abdominal aorta, next to its bifurcation into the common iliac arteries. The caudal mesenteric artery launched branches to the small colon and cranial portion of the rectum. The direct visceral collateral branches of the abdominal aorta were: celiac artery, cranial mesenteric artery, renal arteries and caudal mesenteric artery. The indirect visceral branches were: adrenal arteries and umbilical artery. The external iliac artery emitted the umbilical artery, which irrigated the bladder and continued as a big uterine artery on females, irrigating all the uterus including the ovaries, and on males, the testicular artery, which irrigated the testis.

Key words: abdominal arteries, vascularization, rodents, nutria, Myocastor coypus. 


\section{INTRODUÇÃO}

Os roedores (ordem Rodentia) representam o mais numeroso grupo dentro da classe Mammalia. No Brasil, da família Capromyidae, registra-se a ocorrência apenas do gênero Myocastor, com uma única espécie vivente, o Myocastor coypus, conhecido como ratão-do-banhado, ou nutria [9].

A nutria é um roedor semi-aquático de porte relativamente grande, chega a atingir $60 \mathrm{~cm}$ de comprimento, mais 30 a $40 \mathrm{~cm}$ de cauda, e seu peso, no animal adulto, varia de 6 a $9 \mathrm{~kg}$. Tem a pele recoberta por longos pêlos de coloração geral marrom escuro, dorsalmente, e amarelo claro, ventralmente, muito apreciada pela indústria de peles. Originária do extremo sul da América do Sul, mas disseminada em várias partes do mundo, como EUA e Europa, onde tem sido explorada com fins comerciais, em especial pela qualidade de sua pele e de sua carne. Vivem em banhados, rios e lagos, onde cavam tocas ao longo das margens, as quais são simples túneis que conduzem a um aposento interno que serve de refúgio ou ninho. Alimentam-se de capim, raízes e plantas aquáticas [1].

Informações sobre sua morfologia são raras, e encontram-se alguns relatos sobre o suprimento arterial para as glândulas adrenais [7], sobre a artéria celíaca e seus ramos [8] e sobre a divisão e distribuição das artérias mesentéricas no ratão-do-banhado [6]. Para confrontar os resultados na discussão, utilizamos informações sobre a aorta abdominal em cutias [2] e em cães [3,4].

Este trabalho tem como objetivo sistematizar e descrever os ramos colaterais viscerais da aorta abdominal em Myocastor coypus.

\section{MATERIAIS E MÉTODOS}

Foram utilizadas 30 nutrias (15 fêmeas e 15 machos), jovens e adultas, provenientes de criatório de Caxias do Sul, licenciado pelo IBAMA (Instituto Brasileiro de Meio Ambiente e Recursos Naturais Renováveis). Aos animais foram administradas 5000 UI de heparina (Cristália) e, trinta minutos depois, 20 $\mathrm{ml}$ de Thiopental sódico (Cristália) a 12,5\%, via intraperitoneal. A caixa torácica foi aberta ventralmente em plastrão, a aorta torácica foi canulada próximo ao diafragma e o sistema foi lavado com $250 \mathrm{ml}$ de solução salina. Após, o sistema foi preenchido com látex 603 (Bertoncini), corado em vermelho (BASF) e o animal permaneceu em água corrente por uma

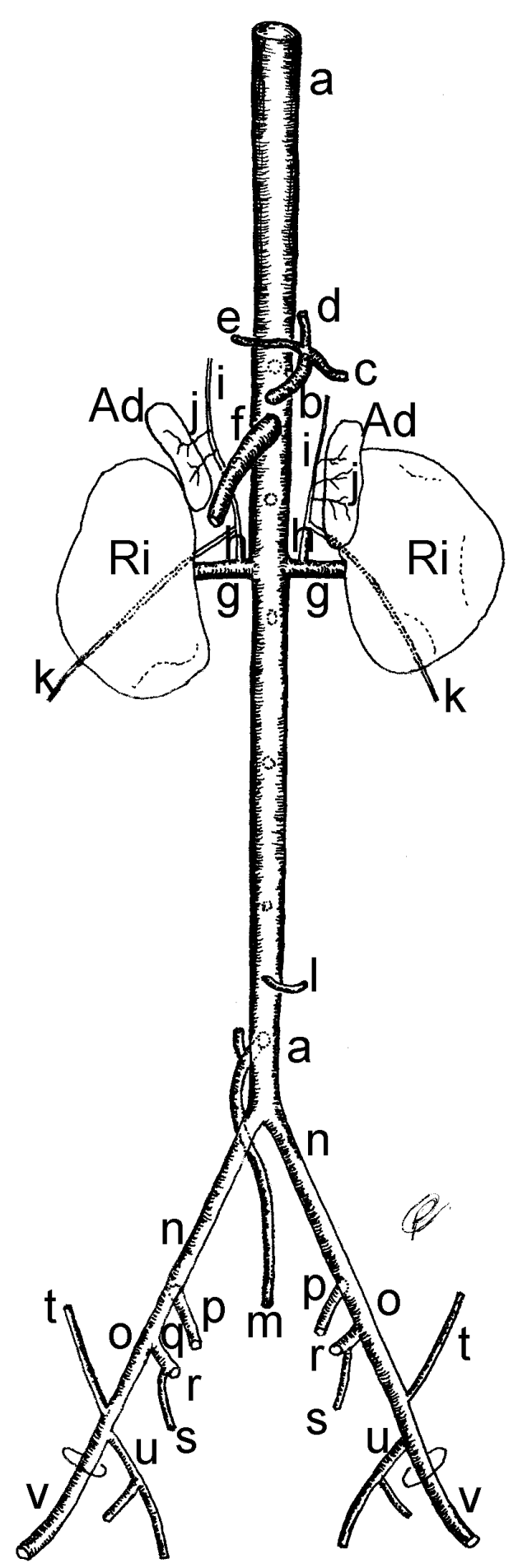

Figura 1. Desenho esquemático em vista ventral da aorta abdominal: a) aorta abdominal; b) a. celíaca; c) a. lienal; d) a. gástrica esquerda; e) a. hepática; f) a. mesentérica cranial; g) a. renal; h) a. frênico-abdominal; i) a. frênica caudal; j) a. adrenal; k) a. abdominal cranial; l) a. mesentérica caudal; m) a. sacral mediana; n) a. ilíaca comum; o) a.ilíaca externa; p) a. ilíaca interna; q) a. umbilical; r) a. vesical; s) a. gonadal; t) a. circunflexa ilíaca profunda; $\mathrm{u}$ ) tronco pudendo epigástrico; v) a. femoral; Ad) glândula adrenal; Ri) rim. 
hora, para solidificação do mesmo. A pele foi rebatida e injetou-se intraperitoneal, $150 \mathrm{ml}$ de formaldeído a $20 \%$ e o animal foi imerso nesta solução por sete dias.

A cavidade abdominal foi aberta na linha mediana ventral e as artérias foram dissecadas, desenhos esquemáticos de todas as preparações foram confeccionados e algumas peças foram fotografadas para documentação. As designações seguiram a Nomina Anatomica Veterinaria (2005) [5].

\section{RESULTADOS}

A aorta, ao atravessar o hiato aórtico do diafragma, emitiu ventralmente seu primeiro ramo colateral visceral, a artéria celíaca. Esta se trifurcou emitindo normalmente as artérias gástrica esquerda, hepática e lienal (Figura 1).

A artéria gástrica esquerda projetou-se em direção ao cárdia do estômago, emitindo ramos esofágicos e distribuindo-se a partir da curvatura menor do estômago em suas faces parietal e visceral.

A artéria hepática dirigiu-se para a cisura portal, lançando as artérias gastroepiplóica direita, pancreaticoduodenal cranial, hepáticas próprias e gástrica direita, irrigando o lado direito do estômago, duodeno, lobo direito do pâncreas e fígado.

A artéria lienal projetou-se ventralmente à esquerda, em direção ao hilo esplênico, emitindo, após um curto tronco, a artéria gastroepiplóica esquerda, para a curvatura maior do estômago no lado esquerdo. A artéria lienal, após lançar ramos gástricos curtos para a parte esquerda do saco gástrico esquerdo, emitiu uma sequiência de artérias lienais próprias ao longo do hilo esplênico.

A artéria mesentérica cranial originou-se da aorta abdominal justaposta e, caudalmente, da origem da artéria celíaca (Figuras 1 e 2). Apresentou normalmente um calibre maior que a da referida artéria, e

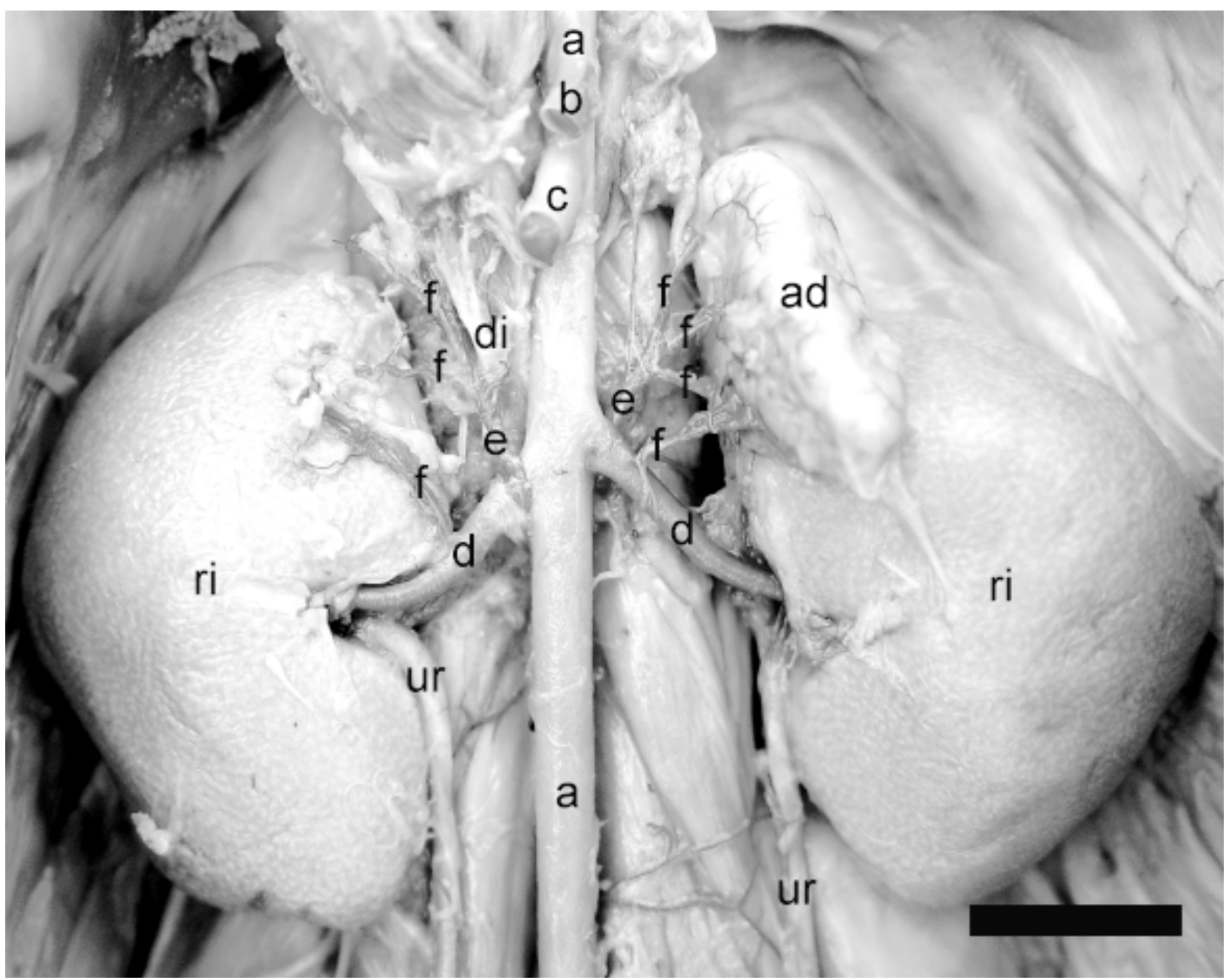

Figura 2. Fotografia em vista ventral (Obs. 16) para salientar os primeiros ramos colaterais viscerais da aorta abdominal: a) aorta abdominal; b) a. celíaca; c) a. mesentérica cranial; d) a. renal; e) a. frênico-abdominal; f) a. adrenal; ad) glândula adrenal; di) pilar direito do diafragma; ri) rim; ur) ureter. Barra $=10 \mathrm{~mm}$. 
emitiu ramos para o trato digestório irrigando as partes caudais do duodeno, lobo direito do pâncreas, jejuno, íleo, ceco, cólon maior e cólon menor.

As artérias renais foram emitidas da aorta abdominal, a alguns centímetros caudais da origem da artéria mesentérica cranial (Figuras 1 e 2), lateralmente e assimétricas em $70 \%$ dos casos e simétricas em 30\%. Nos casos de assimetria, em 13 peças, a artéria renal direita era mais cranial, enquanto em 8 amostras a artéria renal esquerda foi a mais cranial.

A artéria renal mostrou-se única em 96,7\% dos achados e dupla em 3,3\%, tanto à direita como à esquerda. Em um caso à direita, o vaso renal acessório foi mais fino e originado da aorta abdominal cranialmente, enquanto em um outro, à esquerda, o vaso renal acessório era bem mais fino e caudal ao vaso principal.

As glândulas adrenais da nutria apresentaramse extremamente desenvolvidas em relação ao tamanho proporcional do corpo do animal. Sua forma assemelhou-se à de um bastão trifacetado e correspondeu à metade do comprimento, aproximado, de um rim (Figura 2).

As artérias adrenais foram originadas normalmente como ramos colaterais da artéria frênicoabdominal, que era ramo da artéria renal e/ou diretamente da artéria renal (Figuras 1 e 2). A artéria adrenal direita apresentou local de origem, único ou duplo. A artéria adrenal direita apresentou origem única em $76,7 \%$ dos casos, sendo ramo colateral da artéria frênico-abdominal, originada da artéria renal direita, em 17 peças, como ramo da artéria frênica direita, originada da artéria renal direita, em 5 casos, ou como ramo da artéria abdominal direita, originada da artéria renal direita, em um achado. A artéria adrenal direita apresentou origem dupla em 23,3\% das amostras, sendo que, em três achados, a artéria adrenal direita teve origem da artéria frênica caudal, ramo colateral da artéria renal direita, enquanto o segundo vaso teve origem da artéria renal direita. Em dois destes casos, um vaso era ramo colateral da artéria frênico-abdominal direita, originada da artéria renal direita, enquanto o segundo vaso era ramo colateral direto da artéria renal direita. Ainda em duas peças, a artéria adrenal direita originou-se da artéria frênicoabdominal direita, que era ramo colateral da aorta abdominal, enquanto o segundo vaso teve origem direta da artéria renal direita.
A artéria adrenal esquerda apresentou local de origem único ou duplo. A artéria adrenal esquerda apresentou origem única em $33.3 \%$ dos casos, sendo ramo colateral da artéria frênico-abdominal esquerda, originada da artéria renal esquerda, em 8 peças, ou como ramo da artéria frênico-abdominal esquerda, originada da aorta abdominal, em um achado. Já em outra amostra, a artéria adrenal esquerda foi emitida diretamente da aorta abdominal. A artéria adrenal esquerda apresentou duplo local de origem, em $66.7 \%$ das peças, sendo que, em 9 desses casos, o primeiro vaso foi ramo colateral da artéria frênicoabdominal esquerda, originada da artéria renal esquerda e, o segundo vaso, era ramo direto da artéria renal esquerda (Figura 2). Já em 5 peças, o primeiro vaso era ramo colateral da artéria frênica caudal esquerda, ramo da artéria renal esquerda e, o segundo componente, foi ramo direto da artéria renal esquerda. Ainda em 4 achados, o primeiro vaso adrenal era ramo da artéria frênico-abdominal esquerda, ramo direto da aorta abdominal, enquanto o segundo vaso era ramo da artéria renal esquerda. E, em dois achados, o primeiro vaso adrenal era ramo colateral direto da artéria renal esquerda e, o segundo vaso, originou-se da artéria frênico-abdominal esquerda, originada da artéria renal esquerda.

A artéria mesentérica caudal foi emitida da superfície ventral da aorta abdominal, próximo a sua bifurcação em artérias ilíacas comuns direita e esquerda, em 96,7\% dos casos (Figuras 1 e 3), já em $3,3 \%$ ela esteve ausente e seu território foi suprido por um ramo muito desenvolvido da artéria mesentérica cranial, a artéria cólica esquerda. Em 16 casos ela foi emitida cranialmente à origem da artéria sacral mediana, em 8 peças ela foi emitida da aorta abdominal, caudalmente à origem da artéria sacral mediana, já em 5 achados ela foi emitida na mesma altura da origem da artéria sacral mediana. A artéria mesentérica caudal apresentou um fino calibre, bifurcando-se ao aproximar-se da porção distal do cólon menor, em artérias cólica esquerda e retal cranial, irrigando o cólon menor e a porção cranial do reto.

Próximo à entrada da cavidade pélvica, dorsalmente à aorta abdominal, dividiu-se em seus ramos terminais as artérias ilíacas comuns, direita e esquer$\mathrm{da}$, projetadas caudalmente em afastamento divergente de $30^{\circ}$ a $40^{\circ}$ (Figuras 1 e 3). A artéria ilíaca comum dividiu-se em artéria ilíaca interna, continuando-se em 


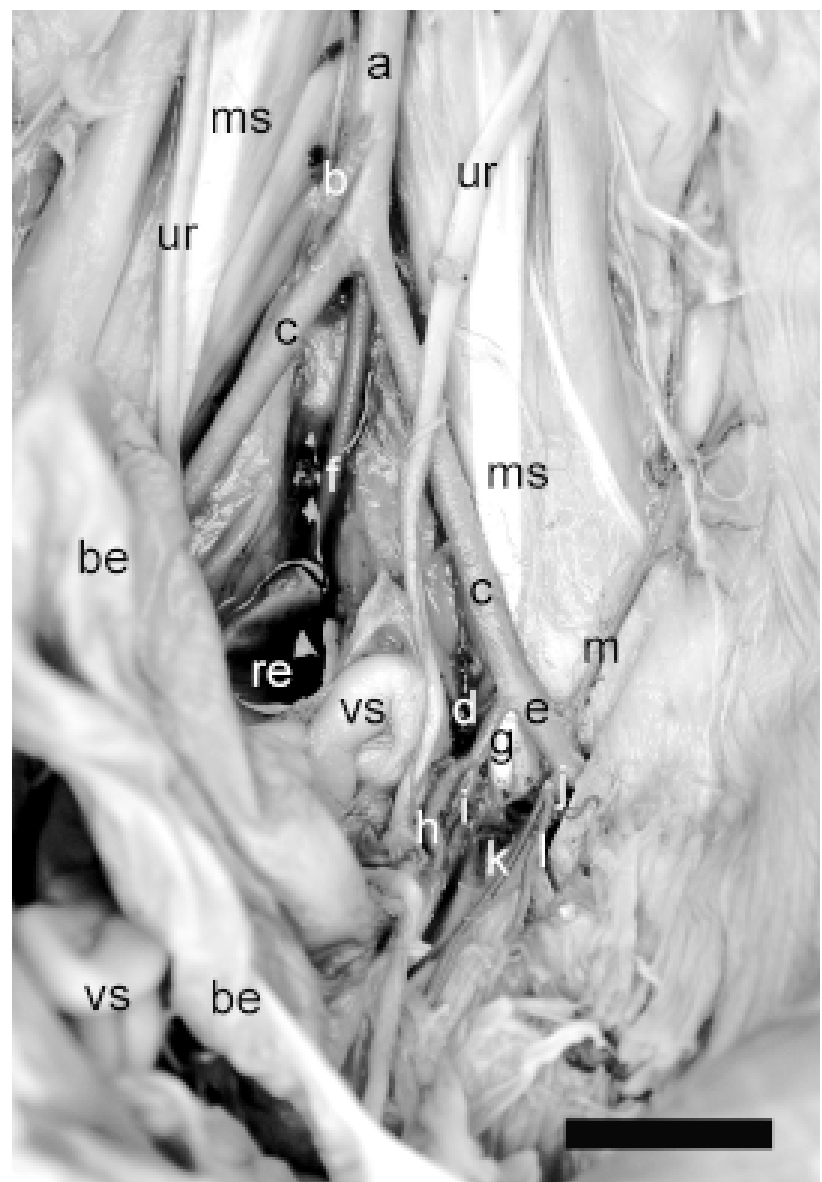

Figura 3. Fotografia em vista ventral (Obs. 16) para salientar os ramos colaterais viscerais da aorta abdominal e seus ramos terminais: a) aorta abdominal; b) a. mesentérica caudal; c) a. ilíaca comum; d) a. ilíaca interna; e) a.ilíaca externa; f) a. sacral mediana; g) a. umbilical; h) a. testicular; i) a. vesical; j) tronco pudendo epigástrico; k) a. pudenda externa; 1) a. epigástrica caudal; m) a. circunflexa ilíaca profunda; ur) ureter; ms) musculatura sublombar; be) bexiga urinária; vs) vesícula seminal; re) reto; Barra $=11 \mathrm{~mm}$.

artéria ilíaca externa, depois de um trajeto de dois a três centímetros, em todas as preparações, nos dois antímeros.

A aorta abdominal, diferentemente dos animais domésticos, emitiu dorsalmente a artéria sacral mediana antes de sua bifurcação (Figuras 1, 3 e 4).

Um último ramo colateral visceral, que não era ramo direto da aorta abdominal e, sim, de seus ramos terminais, as artérias ilíacas externas, foi a artéria umbilical (Figuras 1, 3 e 4). A artéria umbilical direita foi ramo colateral da artéria ilíaca externa direita em $88.7 \%$ dos achados e, em $13.3 \%$ das peças, foi ramo colateral da artéria ilíaca comum direita. A artéria umbilical esquerda foi ramo colateral da artéria ilíaca externa esquerda em $90 \%$ dos casos, ainda foi ramo colateral da artéria ilíaca comum esquerda

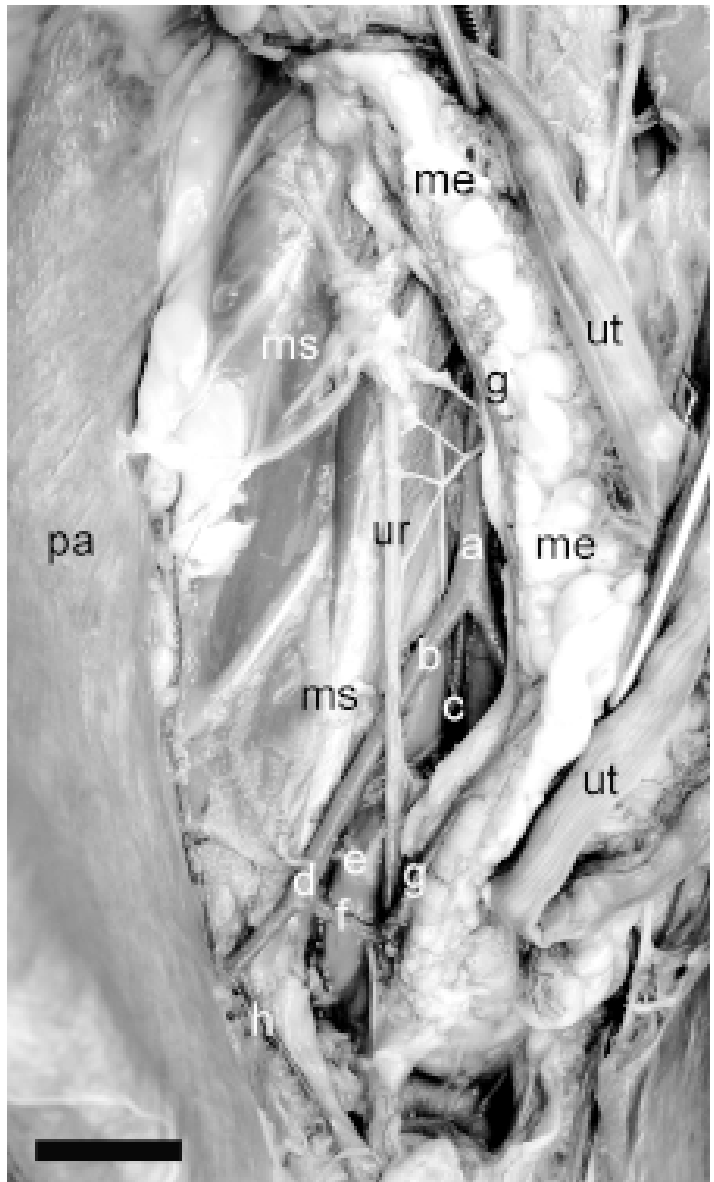

Figura 4. Fotografia em vista ventral (Obs. 02) para salientar a artéria uterina: a) aorta abdominal; b) a. ilíaca comum; c) a. sacral mediana; d) a. ilíaca externa; e) a.ilíaca interna; f) a. umbilical; g) a. uterina; h) tronco pudendo epigástrico; ur) ureter; ms) musculatura sublombar; pa) parede abdominal; ut) corno direito do útero rebatido; Barra $=15 \mathrm{~mm}$.

em $6,7 \%$ das amostras e, em 3,3\%, foi ramo colateral da artéria ilíaca interna esquerda.

A artéria umbilical, um vaso de grosso calibre (Figuras 1, 3 e 4), emitiu caudalmente ramos para a bexiga urinária e, cranialmente, continuou-se como artéria uterina nas fêmeas ou testicular nos machos. A artéria uterina, muito bem desenvolvida, dirigiu-se cranialmente, acompanhando o ligamento largo do útero até alcançar o ovário, irrigando-os (Figura 4). Observou-se em três fêmeas, à direita, a presença de uma artéria ovárica, ramo da artéria renal direita e, em uma fêmea, à esquerda, a artéria ovárica como ramo da artéria renal esquerda. Nos machos, a artéria umbilical também emitiu ramos vesicais craniais, continuando-se ventro-caudalmente, incorporando-se ao funículo espermático como artéria testicular, indo irrigar o testículo e 
o epidídimo. Irrigou também as glândulas anexas do genital masculino (Figura 3). Nos machos, encontrou-se apenas à esquerda em dois casos, a persistência da artéria testicular, como ramo da artéria renal esquerda, a qual alcançou o testículo, contribuindo com a artéria testicular ramo da artéria umbilical, com sua irrigação. Porém, em todas as preparações encontrou-se, tanto nas fêmeas como nos machos, a artéria gonadal ramo da artéria umbilical.

\section{DISCUSSÃO}

A origem da artéria celíaca, na face ventral da aorta abdominal, imediatamente caudal ao hiato aórtico, ocorreu ora separadamente (70\%), ora em tronco comum $(30 \%)$, com a artéria mesentérica cra-nial em 10 nutrias [8]. Em nossos resultados não ocorreu, em nenhum caso, a formação de um tronco comum entre as artérias celíaca e mesentérica cranial. Segundo os mesmos autores, a artéria celíaca dividiu-se para formar as artérias lienal, gástrica esquerda e hepática, resultado este coincidente com nossos achados.

No cão, a artéria celíaca, ímpar, foi um curto vaso que surgiu da face ventral da aorta abdominal, ao nível do hiato aórtico do diafragma. Normalmente, dividiu-se nas artérias gástrica esquerda, hepática e lienal [3,4]. A artéria mesentérica cranial, ímpar, surgiu bem próxima e caudalmente à artéria celíaca, da superfície ventral da aorta abdominal. Ela supre principalmente à metade caudal do duodeno, até a parte cranial do cólon descendente [4]. Esta origem e distribuição também foi observada na nutria.

Em cutias adultas, a artéria mesentérica caudal bifurcou-se, numa artéria cólica esquerda e numa artéria retal cranial [2]. No cão, a artéria mesentérica caudal, ímpar, é pequena e surgiu ventralmente da aorta abdominal. Ela supriu as partes média e caudal do cólon descendente e a parte cranial do reto. Após percurso de cerca de 5 centímetros, ela dividiu-se nas artérias cólica esquerda e retal cranial [4]. Esta disposição da artéria mesentérica caudal também foi encontrada na nutria.

As artérias renais, em cães, foram pares e surgiram assimetricamente das superfícies laterais da aorta abdominal. A artéria renal direita era de localização um tanto cranial à artéria esquerda correspondente [4]. Em nossos resultados, as artérias renais geralmente foram assimétricas e, nestes casos, normalmente, a artéria renal direita foi mais cranial.

As artérias adrenais, em nutrias, diferiam das descrições já realizadas em outros roedores e lagomorfas. Para a glândula adrenal direita destinaram-se ramos provenientes das artérias frênica caudal direita, abdominal cranial direita, renal direita, primeira artéria lombar e aorta abdominal. Já para a glândula adrenal esquerda foram emitidos ramos oriundos das artérias renal esquerda, aorta abdominal, abdominal cranial esquerda e segunda artéria lombar [7]. Em nossos achados ocorreram muitas variações, porém os vasos adrenais eram mais freqüentemente originados do tronco frênico-abdominal, que era ramo da artéria renal, tanto à direita como à esquerda.

Em cão, as artérias testiculares ou ovarianas, pares, foram vasos pequenos, emitidos ventrolateralmente da aorta abdominal, aproximadamente a meio caminho entre as artérias renais e mesentérica caudal [4]. Em nossos achados em nutria, esta artéria não foi identificada como ramo colateral da aorta abdominal.

A artéria umbilical em cães surgiu da artéria ilíaca interna, próximo a sua origem ou da parte final da artéria aorta abdominal. Seu segmento proximal é normalmente patente, enquanto em seu segmento distal, o lúmem, está obliterado, formando o ligamento redondo da bexiga urinária [4]. Na nutria, a artéria umbilical, tanto no macho como na fêmea, assumiu o território das artérias gonadais. Em gambá, a artéria umbilical foi normalmente ramo colateral da artéria ilíaca comum [2], enquanto em nossos resultados ela foi normalmente ramo da artéria ilíaca externa.

\section{CONCLUSÕES}

Com base nos resultados do presente trabalho, podemos concluir que, os ramos colaterais viscerais diretos da aorta abdominal, são: tronco celíaco, artéria mesentérica cranial, artérias renal direita e esquerda e artéria mesentérica caudal. Os ramos colaterais viscerais indiretos são: artérias adrenais e artéria umbilical. A artéria umbilical, um grande ramo colateral visceral, irriga a bexiga urinária e continua como uma grande artéria uterina, nas fêmeas, irrigando todo o útero e ovário e, nos machos, a artéria testicular, que irrigou o testículo.

\section{REFERÊNCIAS}

1 Baroffio R.A., De Paolii J.C. \& Fiordelisi A.O. 1979. Nuestra Nutria. 2nd edn. Buenos Aires: Editorial Hemisferio Sur S.A., $159 \mathrm{p}$. 
2 Carvalho M.A.M., Miglino M.A., Didio L.J.A. \& Melo A.P.F. 1999. Artérias mesentéricas cranial e caudal em cutias (Dasyprocta aguti). Veterinária Notícias. 2: 17-24.

3 Evans H.E. 1993. The heart and the arteries. In: Evans H.E. (Ed). Miller's Anatomy of the dog. 3rd edn. Philadelphia: W.B. Saunders Company, pp. 586-681.

4 Ghoshal N.G. 1986. Coração e artérias do carnívoro. In: Getty R. (Ed). Anatomia dos animais domésticos. 5. ed. Rio de Janeiro: Interamericana, pp. 1497-1550.

5 International Committee on Veterinary Gross Anatomical Nomenclature. 2005. Nomina Anatomica Veterinaria. 5th edn. New York: Nomina Anatomica Veterinaria, 198p.

6 Machado G.V., Gonçalves P.R., Parizzi A. \& Souza J.R. 2006. Padrão de divisão e distribuição das artérias mesentéricas no ratão-do-banhado (Myocastor coypus - Rodentia: Mammalia). Biotemas. 1: 59-63.

7 Machado G.V., Romagnolli P., Souza J.R., Turquetti V.S., Uliana S.M. \& Silva M.H. 2002. Suprimento arterial para as glândulas adrenais no ratão-do-banhado (Myocastor coypus Molina, 1782). Archives of Veterinary Science. 2: 9-14.

8 Machado G.V., Souza J.R., Gonçalves P.R., Parizzi A. \& Donin D.G. 2002. A artéria celíaca e seus ramos no ratão-dobanhado (Myocastor coypus - Rodentia: Mammalia). Biotemas. 2: 41-54.

9 Silva F. 1994. Mamíferos silvestres do Rio Grande do Sul. Porto Alegre: Fundação Zoobotânica do Rio Grande do Sul, 282p. 